PROCEEDINGS OF THE

AMERICAN MATHEMATICAL SOCIETY

Volume 133, Number 12, Pages 3581-3587

S 0002-9939(05)07923-2

Article electronically published on June 8, 2005

\title{
SYMMETRIC STABLE PROCESSES IN PARABOLA-SHAPED REGIONS
}

\author{
RODRIGO BAÑUELOS AND KRZYSZTOF BOGDAN
}

(Communicated by Richard C. Bradley)

\begin{abstract}
We identify the critical exponent of integrability of the first exit time of the rotation invariant stable Lévy process from a parabola-shaped region.
\end{abstract}

\section{INTRODUCTION}

For $d=2,3, \ldots$ and $0<\beta<1$, we define the parabola-shaped region in $\mathbb{R}^{d}$ as

$$
\mathcal{P}_{\beta}=\left\{x=\left(x_{1}, \tilde{x}\right): x_{1}>0, \tilde{x} \in \mathbb{R}^{d-1},|\tilde{x}|<x_{1}^{\beta}\right\} .
$$

Let $0<\alpha<2$. By $\left\{X_{t}\right\}$ we denote the isotropic $\alpha$-stable $\mathbb{R}^{d}$-valued Lévy process ([17]). The process is time-homogeneous, has right-continuous trajectories with left limits, $\alpha$-stable rotation invariant independent increments, and characteristic function

$$
\mathbf{E}_{x} e^{i \xi\left(X_{t}-x\right)}=e^{-t|\xi|^{\alpha}}, \quad x \in \mathbb{R}^{d}, \quad \xi \in \mathbb{R}^{d}, \quad t \geq 0 .
$$

Here $\mathbf{E}_{x}$ is the expectation with respect to the distribution $\mathbf{P}_{x}$ of the process starting from $x \in \mathbb{R}^{d}$. For an open set $U \subset \mathbb{R}^{d}$, we define $\tau_{U}=\inf \left\{t \geq 0 ; X_{t} \notin U\right\}$, the first exit time of $U$ ([17]). In the case of the parabola-shaped region $\mathcal{P}_{\beta}$, we simply write $\tau_{\beta}$ for $\tau_{\mathcal{P}_{\beta}}$.

The main result of this note is the following result.

Theorem 1. Let $p \geq 0$. Then $E_{x} \tau_{\beta}^{p}<\infty$ for some (hence for all) $x \in \mathcal{P}_{\beta}$ if and only if $p<p_{0}$, where

$$
p_{0}=\frac{(d-1)(1-\beta)+\alpha}{\alpha \beta} .
$$

Theorem 1 may be regarded as an addition to the research direction initiated in [3], where it was proved that for $\beta=1 / 2, d=2$ and $\left\{X_{t}\right\}$ replaced by the Brownian motion process $\left\{B_{t}\right\}, \tau_{\beta}$ is subexponentially integrable. For $\left\{B_{t}\right\}$, the generalizations to all the considered domains $\mathcal{P}_{\beta}$ (along with essential strengthening of the result of [3) were subsequently obtained in [14, 15, [5, 11] and 2]. We should note that this direction of research was influenced to a large degree by the

Received by the editors June 14, 2004 and, in revised form, July 14, 2004.

2000 Mathematics Subject Classification. Primary 31B05, $60 \mathrm{~J} 45$.

Key words and phrases. Symmetric stable process, parabola, exit time, harmonic measure.

The first author was supported in part by NSF grant \# 9700585-DMS.

The second author was supported in part by KBN (2P03A 04122 ) and by RTN (HPRN-CT2001-00273-HARP).

(C)2005 American Mathematical Society Reverts to public domain 28 years from publication 
result of Burkholder [10] on the critical order of integrability of the exit times of $\left\{B_{t}\right\}$ from cones. For more on the many generalizations of Burkholder's result we refer the reader to [4] and references therein.

Brownian motion is a limiting case of the isotropic $\alpha$-stable process and $B_{2 t}$ corresponds to $\alpha=2$ via the analogue of (11). Extension of some of the abovementioned results pertaining to cones to the case $0<\alpha<2$ (that is, for $\left\{X_{t}\right\}$ ) were given in [12, 13, [16] and [1; see also [7. It should be emphasized that while there are many similarities between $\left\{X_{t}\right\}$ and $\left\{B_{2 t}\right\}$, there also exist essential differences in their respective properties and their proofs. For example the critical integrability of the exit time of $\left\{X_{t}\right\}$ is less than 1 for every cone, however narrow it may be ([1]), while it is arbitrarily large for $\left\{B_{t}\right\}$ in sufficiently narrow cones ([10]). A similar remark applies to regions $\mathcal{P}_{\beta}$. The critical exponent of integrability of $\tau_{\beta}$ for $\left\{X_{t}\right\}$ given in Theorem [1 is qualitatively different from that of $\left\{B_{t}\right\}$ ([2]).

Finally, we have recently learned that Pedro J. Méndez-Hernández, in a paper in preparation, has obtained results similar to those presented. His results apply to more general regions defined by other "slowly" increasing functions.

The proof of Theorem 1 is based on estimates for harmonic measure of $\left\{X_{t}\right\}$, following the general idea used in 2 for $\left\{B_{t}\right\}$. The necessity of the condition $p<p_{0}$ for finiteness of $E_{x} \tau_{\beta}^{p}$ is proved in Lemma 2 and the sufficiency is proved in Lemma 6. The main technical result of the paper is Lemma 5 , where we prove sharp estimates for the harmonic measure of cut-offs of the parabola-shaped region. At the end of the paper we discuss some remaining open problems.

\section{Proofs}

We begin by reviewing the notation. By $|\cdot|$ we denote the Euclidean norm in $\mathbb{R}^{d}$. For $x \in \mathbb{R}^{d}, r>0$, and a set $A \subset \mathbb{R}^{d}$ we let $B(x, r)=\left\{y \in \mathbb{R}^{d}:|x-y|<r\right\}$ and $\operatorname{dist}(A, x)=\inf \{|x-y|: y \in A\} . \quad A^{c}$ is the complement of $A$. We always assume Borel measurability of the considered sets and functions. In equalities and inequalities, unless stated otherwise, $c$ denotes some unspecified positive real number whose value depends only on $d, \alpha$ and $\beta$.

It is well known that $\left(X_{t}, \mathbf{P}_{x}\right)$ is a strong Markov process with respect to the standard filtration ([17]).

For an open set $U \subset \mathbb{R}^{d}$, with exit time $\tau_{U}$, the $\mathbf{P}_{x}$ distribution of $X_{\tau_{U}}$,

$$
\omega_{U}^{x}(A):=P_{x}\left\{X_{\tau_{U}} \in A, \tau_{U}<+\infty\right\}, \quad A \subset \mathbb{R}^{d},
$$

is a subprobability measure concentrated on $U^{c}$ (probability measure if $U$ is bounded), called the harmonic measure. When $r>0,|x|<r$ and $B=B(0, r) \subset$ $\mathbb{R}^{d}$, the corresponding harmonic measure has density function $P_{r}(x, y)=d \omega_{B}^{x} / d y$ (the Poisson kernel for the ball). We have

$$
P_{r}(x, y)=C(d, \alpha)\left[\frac{r^{2}-|x|^{2}}{|y|^{2}-r^{2}}\right]^{\alpha / 2}|y-x|^{-d} \quad \text { if } \quad|y|>r,
$$

where $C(d, \alpha)=\Gamma(d / 2) \pi^{-d / 2-1} \sin (\pi \alpha / 2)$, and $P_{r}(x, y)=0$ otherwise $([\underline{6})$.

The scaling property of $X_{t}$ plays a role in this paper. Namely, for every $r>0$ and $x \in \mathbb{R}^{d}$ the $\mathbf{P}_{x}$ distribution of $\left\{X_{t}\right\}$ is the same as the $\mathbf{P}_{r x}$ distribution of $\left\{r^{-1} X_{r^{\alpha} t}\right\}$ (see (10). In particular, the $\mathbf{P}_{x}$ distribution of $\tau_{U}$ is the same as the $\mathbf{P}_{r x}$ distribution of $r^{-\alpha} \tau_{r U}$. In short, $\tau_{r U}=r^{\alpha} \tau_{U}$ in distribution. 
Let $\mathcal{P}_{\beta}{ }^{\prime}=\mathcal{P}_{\beta} \cap\left\{x_{1}>1,|\tilde{x}|<x_{1}^{\beta} / 2\right\}$. We claim that if $x \in \mathcal{P}_{\beta}{ }^{\prime}$, then $B\left(x,|x|^{\beta} / 5\right)$ $\subset \mathcal{P}_{\beta}$. Indeed, let $x=\left(x_{1}, \tilde{x}\right) \in \mathcal{P}_{\beta}{ }^{\prime}$ and $y=\left(y_{1}, \tilde{y}\right) \in B\left(x,|x|^{\beta} / 5\right)$. We have $|\tilde{x}|<x_{1}^{\beta} / 2<x_{1} / 2$, hence

$$
1<x_{1} \leq|x|=\sqrt{x_{1}^{2}+|\tilde{x}|^{2}}<\sqrt{5 / 4} x_{1}<5 x_{1} / 4 .
$$

Then

$$
y_{1} \geq x_{1}-|x|^{\beta} / 5>x_{1}-|x| / 5>x_{1}-(5 / 4) x_{1} / 5=3 x_{1} / 4,
$$

and so

$$
|\tilde{y}| \leq|\tilde{x}|+|x|^{\beta} / 5<x_{1}^{\beta} / 2+\left(5 x_{1} / 4\right)^{\beta} / 5<3 x_{1}^{\beta} / 4<3\left(4 y_{1} / 3\right)^{\beta} / 4<y_{1}^{\beta},
$$

which yields $y \in \mathcal{P}_{\beta}$, as claimed.

Lemma 2. If $p \geq p_{0}$, then $E_{x} \tau_{\beta}^{p}=\infty$ for every $x \in \mathcal{P}_{\beta}$.

Proof. Define $\tau=\inf \left\{t \geq 0: X_{t} \notin B\left(X_{0},\left|X_{0}\right|^{\beta} / 5\right)\right\}$. For $y \in \mathbb{R}^{d}$ and (nonnegative) $p$ by scaling, we have $\mathbf{E}_{y} \tau^{p}=\mathbf{E}_{0} \tau_{B(0,1)}^{p}\left(|y|^{\beta} / 5\right)^{p \alpha}=c|y|^{\alpha \beta p}$. Let $x \in \mathcal{P}_{\beta}, r=$ $\operatorname{dist}\left(x, \mathcal{P}_{\beta}{ }^{c}\right), B=B(x, r)$, and let $R$ be so large that $B \subset \mathcal{P}_{\beta} \cap\left\{y_{1} \leq R\right\}$. By strong Markov property we have

$$
\begin{aligned}
& \mathbf{E}_{x} \tau_{\beta}{ }^{p} \geq \mathbf{E}_{x}\left\{X_{\tau_{B}} \in \mathcal{P}_{\beta}{ }^{\prime} ; \mathbf{E}_{X_{\tau_{B}}} \tau_{\beta}{ }^{p}\right\} \geq \mathbf{E}_{x}\left\{X_{\tau_{B}} \in \mathcal{P}_{\beta}{ }^{\prime} ; \mathbf{E}_{X_{\tau_{B}}} \tau^{p}\right\} \\
& =c \mathbf{E}_{x}\left\{X_{\tau_{B}} \in \mathcal{P}_{\beta^{\prime}} ;\left|X_{\tau_{B}}\right|^{\alpha \beta p}\right\} \geq c \int_{\mathcal{P}_{\beta^{\prime}} \cap\left\{y_{1}>R\right\}} P_{r}(0, y-x)|y|^{\alpha \beta p} d y \\
& \geq \operatorname{cr}^{\alpha} C(d, \alpha) \int_{R}^{\infty} d y_{1} \int_{|\tilde{y}|<y_{1}^{\beta} / 2}|y-x|^{-d-\alpha}|y|^{\alpha \beta p} d \tilde{y} \\
& \geq \text { const. } \int_{R}^{\infty} y_{1}^{\beta(d-1)+\alpha \beta p-d-\alpha} d y_{1} \text {. }
\end{aligned}
$$

If $p \geq p_{0}$, then $\beta(d-1)+\alpha \beta p-d-\alpha \geq \beta(d-1)+(d-1)(1-\beta)+\alpha-d-\alpha=-1$ and the last integral is positive infinity, proving the lemma.

For $0 \leq u<v \leq \infty$, we let $\mathcal{P}_{\beta}{ }^{u, v}=\mathcal{P}_{\beta} \cap\left\{\left(x_{1}, \tilde{x}\right): u \leq x_{1}<v\right\}$. Let $0<s<\infty$ and define $\tau_{s}=\tau(s)=\tau_{\mathcal{P}_{\beta}}{ }^{0, s}$. Consider the cylinder

$$
\mathcal{C}=\left\{x=\left(x_{1}, \tilde{x}\right) \in \mathbb{R}^{d}:-\infty<x_{1}<s,|\tilde{x}|<s^{\beta}\right\} .
$$

Clearly for $A \subset \mathcal{P}_{\beta}$,

$$
\mathbf{P}_{x}\left\{X_{\tau_{s}} \in A\right\} \leq \mathbf{P}_{x}\left\{X_{\tau_{\mathcal{C}}} \in A\right\}, \quad x \in \mathbb{R}^{d} .
$$

By Lemma 4.3 of $[9]$ for $A \subset \mathbb{R}^{d} \cap\left\{z_{1} \geq s\right\}$ we have

$$
\mathbf{P}_{x}\left\{X_{\tau_{\mathcal{C}}} \in A\right\} \leq c \int_{z=\left(z_{1}, \tilde{z}\right) \in A} \frac{s^{\alpha \beta}}{|z-x|^{d+\alpha}}\left[\frac{s^{\alpha \beta / 2}}{\left(z_{1}-s\right)^{\alpha / 2}} \vee 1\right] d z, \quad x \in \mathcal{C} .
$$

The following lemma will simplify the use of the estimate (5).

Lemma 3. Let $s \geq 1$ and $x=\left(x_{1}, \tilde{x}\right) \in \mathcal{P}_{\beta}$ with $x_{1} \leq s / 2$. Let $s \leq u<v \leq \infty$ and assume that either $u \geq s+s^{\beta}$ or $u=s$ and $v \geq s+s^{\beta}$. Then

$$
\mathbf{P}_{x}\left\{X_{\tau_{s}} \in \mathcal{P}_{\beta}^{u, v}\right\} \leq c s^{\alpha \beta} \int_{u}^{v} t^{-\alpha \beta p_{0}-1} d t .
$$


Proof. Denote

$$
f(y)=\frac{d \omega_{\mathcal{P}_{\beta} 0, s}^{x}}{d y}(y) .
$$

Let $y=\left(y_{1}, \tilde{y}\right) \in \mathcal{P}_{\beta}{ }^{s, \infty}$. From (4) and (5D) we can conclude that

$$
f(y) \leq c \frac{s^{\alpha \beta}}{|y-x|^{d+\alpha}}\left[\frac{s^{\alpha \beta / 2}}{\left(y_{1}-s\right)^{\alpha / 2}} \vee 1\right] .
$$

Since $s \geq 1$, we have $|\tilde{y}| \leq y_{1}$ and $|y| \leq \sqrt{2} y_{1}$. As $|y-x| \geq y_{1}-x_{1} \geq y_{1} / 2$,

$$
f(y) \leq c s^{\alpha \beta} y_{1}^{-d-\alpha}\left[\frac{s^{\alpha \beta / 2}}{\left(y_{1}-s\right)^{\alpha / 2}} \vee 1\right] .
$$

If $u \geq s+s^{\beta}$, then

$$
\begin{aligned}
\mathbf{P}_{x}\left\{X_{\tau_{s}} \in \mathcal{P}_{\beta}{ }^{u, v}\right\} & \leq c s^{\alpha \beta} \int_{u}^{v} y_{1}^{-d-\alpha} \int_{|\tilde{y}|<y_{1}^{\beta}} d \tilde{y} d y_{1} \\
& =c s^{\alpha \beta} \int_{u}^{v} t^{-d-\alpha+\beta(d-1)} d t=c s^{\alpha \beta} \int_{u}^{v} t^{-\alpha \beta p_{0}-1} d t .
\end{aligned}
$$

If $u=s$ and $v \geq s+s^{\beta}$, we consider

$$
I(s)=s^{\alpha \beta} \int_{s}^{s+s^{\beta}} t^{-\alpha \beta p_{0}-1} d t
$$

and

$$
I I(s)=s^{\alpha \beta} \int_{s}^{s+s^{\beta}} t^{-\alpha \beta p_{0}-1} \frac{s^{\alpha \beta / 2}}{(t-s)^{\alpha / 2}} d t .
$$

It is enough to verify that $I I(s) \leq c I(s)$. Note that $s+s^{\beta} \leq 2 s$, hence

$$
I(s) \geq s^{\alpha \beta}(2 s)^{-\alpha \beta p_{0}-1} s^{\beta}=c s^{-\alpha \beta p_{0}+\alpha \beta+\beta-1} .
$$

Since

$$
I I(s) \leq s^{\alpha \beta} s^{-\alpha \beta p_{0}-1} s^{\alpha \beta / 2} \int_{0}^{s^{\beta}} z^{-\alpha / 2} d z=c s^{-\alpha \beta p_{0}+\alpha \beta+\beta-1},
$$

we get $I I(s) \leq c I(s)$.

The following result is an immediate consequence of Lemma 3 ,

Lemma 4. If $s \geq 1, x=\left(x_{1}, \tilde{x}\right) \in \mathbb{R}^{d}$ and $x_{1} \leq s / 2$, then

$$
\mathbf{P}_{x}\left\{X_{\tau_{s}} \in \mathcal{P}_{\beta}\right\} \leq c s^{-\alpha \beta\left(p_{0}-1\right)} .
$$

Estimate (7) is sharp if, for example, $x=(s / 2, \tilde{0})$, where $\tilde{0}=(0, \ldots, 0) \in \mathbb{R}^{d-1}$. Indeed, let $B=B\left(x, c s^{\beta}\right) \subset \mathcal{P}_{\beta}^{0, s}$, where $c>0$ is small enough. By (2) we have

$$
\begin{aligned}
\mathbf{P}_{x}\left\{X_{\tau_{s}} \in \mathcal{P}_{\beta}\right\} & \geq \mathbf{P}_{x}\left\{X_{\tau_{B}} \in \mathcal{P}_{\beta}^{s, \infty}\right\} \\
& \geq c\left(s^{\beta}\right)^{\alpha} \int_{s}^{\infty} d z_{1} \int_{\left\{\tilde{z} \in \mathbb{R}^{d-1}:|\tilde{z}|<z_{1}^{\beta}\right\}}\left|\left(z_{1}, \tilde{z}\right)-x\right|^{-d-\alpha} d \tilde{z} \\
& \geq c s^{\alpha \beta} \int_{s}^{\infty} t^{\beta(d-1)-d-\alpha} d t=c s^{-\alpha \beta\left(p_{0}-1\right)} .
\end{aligned}
$$

We can, however, improve the estimate when $x$ is small relative to $s$.

Lemma 5. If $s \geq 1, x=\left(x_{1}, \tilde{x}\right) \in \mathbb{R}^{d}$ and $x_{1} \leq s / 2$, then

$$
\mathbf{P}_{x}\left\{X_{\tau_{s}} \in \mathcal{P}_{\beta}\right\} \leq C\left(x_{1} \vee 1\right)^{\alpha \beta} s^{-\alpha \beta p_{0}} .
$$


Proof. If $s \leq S$, where $0<S<\infty$ is fixed, then (9) trivially holds with $C=S^{\alpha \beta p_{0}}$. Hence from now on we assume $s \geq S$, where $S=2^{k_{0}}$ and $k_{0} \geq 2$ is such that

$$
\frac{8^{\alpha \beta} c}{\alpha \beta\left(p_{0}-1\right)}\left(2^{k_{0}-1}\right)^{-\alpha \beta\left(p_{0}-1\right)}<\frac{1}{2} .
$$

The reason for this choice of $k_{0}$ will be made clear later on. Here and in what follows $c$ is the constant of Lemma 3. We will prove (9) by induction. If $s / 4 \leq x_{1} \leq s / 2$, then by Lemma 4

$$
\mathbf{P}_{x}\left\{X_{\tau_{s}} \in \mathcal{P}_{\beta}\right\} \leq c_{1} s^{\alpha \beta} s^{-\alpha \beta p_{0}} \leq 4^{\alpha \beta} c_{1} x_{1}^{\alpha \beta} s^{-\alpha \beta p_{0}}=c_{1}\left(x_{1} \vee 1\right)^{\alpha \beta} s^{-\alpha \beta p_{0}} .
$$

Assume that $n$ is a natural number and (9) holds for all $x=\left(x_{1}, \tilde{x}\right) \in \mathcal{P}_{\beta}$ such that $s / 2^{n+1} \leq x_{1} \leq s / 2$.

Let $s / 2^{n} \geq 1$ and $x=\left(x_{1}, \tilde{x}\right) \in \mathcal{P}_{\beta}$ be such that $s / 2^{n+2} \leq x_{1}<s / 2^{n+1}$. Note that $1 \leq s / 2^{n} \leq 4 x_{1}$ here. We have

$$
\begin{aligned}
\mathbf{P}_{x}\left\{X_{\tau_{s}} \in \mathcal{P}_{\beta}\right\} & \leq \mathbf{P}_{x}\left\{X_{\tau\left(s / 2^{n}\right)} \in \mathcal{P}_{\beta}^{s / 2, \infty}\right\} \\
& +\mathbf{E}_{x}\left\{X_{\tau\left(s / 2^{n}\right)} \in \mathcal{P}_{\beta}^{s / 2^{n}, s / 2} ; \mathbf{P}_{X_{\tau\left(s / 2^{n}\right)}}\left\{X_{\tau_{s}} \in \mathcal{P}_{\beta}\right\}\right\} \\
& =I+I I .
\end{aligned}
$$

By Lemma 3 ,

$$
\begin{aligned}
I & \leq c\left(s / 2^{n}\right)^{\alpha \beta} \int_{s / 2}^{\infty} t^{-\alpha \beta p_{0}-1} d t=c 2^{\alpha \beta p_{0}}\left(s / 2^{n}\right)^{\alpha \beta} s^{-\alpha \beta p_{0}} \\
& \leq c 2^{\alpha \beta p_{0}+2 \alpha} x_{1}^{\alpha \beta} s^{-\alpha \beta p_{0}} \leq c_{2}\left(x_{1} \vee 1\right)^{\alpha \beta} s^{-\alpha \beta p_{0}}
\end{aligned}
$$

By Lemma 3 and induction

$$
\begin{aligned}
I I & \leq 2^{\alpha \beta} c\left(s / 2^{n}\right)^{\alpha \beta} \int_{s / 2^{n}}^{s / 2} t^{-\alpha \beta p_{0}-1} C t^{\alpha \beta} s^{-\alpha \beta p_{0}} d t \\
& \leq \frac{2^{\alpha \beta} c}{\alpha \beta\left(p_{0}-1\right)}\left(s / 2^{n}\right)^{-\alpha \beta\left(p_{0}-1\right)} C\left(s / 2^{n}\right)^{\alpha \beta} s^{-\alpha \beta p_{0}} \\
& \leq \frac{8^{\alpha \beta} c}{\alpha \beta\left(p_{0}-1\right)}\left(s / 2^{n}\right)^{-\alpha \beta\left(p_{0}-1\right)} C\left(x_{1} \vee 1\right)^{\alpha \beta} s^{-\alpha \beta p_{0}}
\end{aligned}
$$

Thus

$$
R=\frac{I+I I}{\left(x_{1} \vee 1\right)^{\alpha \beta} s^{-\alpha \beta p_{0}}} \leq c_{2}+\frac{8^{\alpha \beta} c}{\alpha \beta\left(p_{0}-1\right)}\left(s / 2^{n}\right)^{-\alpha \beta\left(p_{0}-1\right)} C .
$$

For $n$ such that $s / 2^{n} \geq 2^{k_{0}}$ we have $R \leq c_{2}+C / 2$ and we can take $C=2\left(c_{1} \vee c_{2}\right)$ in our inductive assumption to the effect that $R \leq c_{2}+c_{1} \vee c_{2} \leq C$. Therefore, (9) holds for every $x=\left(x_{1}, \tilde{x}\right) \in \mathcal{P}_{\beta}$, satisfying $s / 2^{n+2} \leq x_{1} \leq s / 2$.

By induction, (9) is true with $C=2\left(c_{1} \vee c_{2}\right)$ for all $x=\left(x_{1}, \tilde{x}\right) \in \mathcal{P}_{\beta}$, satisfying $2^{k_{0}-2} \leq x_{1} \leq s / 2$. 


$$
\begin{aligned}
& \text { If } x=\left(x_{1}, \tilde{x}\right) \in \mathcal{P}_{\beta} \text { and } 0<x_{1} \leq 2^{k_{0}-2} \text {, then } \\
& \mathbf{P}_{x}\left\{X_{\tau_{s}} \in \mathcal{P}_{\beta}\right\} \leq \mathbf{P}_{x}\left\{X_{\tau\left(2^{k_{0}} / 2\right)} \in \mathcal{P}_{\beta}{ }^{s / 2, \infty}\right\} \\
& +\mathbf{E}_{x}\left\{X_{\tau\left(2^{\left.k_{0} / 2\right)}\right.} \in \mathcal{P}_{\beta} 2^{2^{k_{0}} / 2, s / 2} ; \mathbf{P}_{X_{\tau\left(2^{\left.k_{0} / 2\right)}\right.}}\left\{X_{\tau_{s}} \in \mathcal{P}_{\beta}\right\}\right\} \\
& \leq c\left(2^{k_{0}} / 2\right)^{\alpha \beta} \int_{s / 2}^{\infty} t^{-\alpha \beta p_{0}-1} d t \\
& +2^{\alpha \beta} c\left(2^{k_{0}} / 2\right)^{\alpha \beta} \int_{2^{k_{0} / 2}}^{s / 2} t^{-\alpha \beta p_{0}-1} 2\left(c_{1} \vee c_{2}\right) t^{\alpha \beta} s^{-\alpha \beta p_{0}} d t \\
& \leq c_{3} s^{-\alpha \beta p_{0}} \leq c_{3}\left(x_{1} \vee 1\right)^{\alpha \beta} s^{-\alpha \beta p_{0}} .
\end{aligned}
$$

We used here our previous estimates. The proof is complete.

Note that (9) is sharp if, for example, $x=\left(x_{1}, \tilde{0}\right)$ and $1 \leq x_{1} \leq s / 2$. This can be verified as in (8) to the effect that for such $x$ the upper bound in Lemma 5 is of the same order as $\mathbf{P}_{x}\left\{X_{\tau_{B}} \in \mathcal{P}_{\beta}{ }^{s, \infty}\right\}$, where $B$ is the largest ball centered at $x$ and such that $B \subset \mathcal{P}_{\beta}$.

Informally, $\left\{X_{t}\right\}$ goes to $\mathcal{P}_{\beta}^{s, \infty}$ "mostly" by a direct jump from $B$.

This informal rule seems to be related to a "thinness" of $\mathcal{P}_{\beta}$ at infinity (or its inversion 8 at 0 ). Such a rule is false for cones (1]).

Lemma 6. If $p<p_{0}$, then $E_{x} \tau_{\beta}{ }^{p}<\infty$ for every $x \in \mathcal{P}_{\beta}$.

Proof. Let $1 / \lambda_{1}$ be the first eigenvalue of the Green operator

$$
G_{B} f(\tilde{x})=\mathcal{E}_{\tilde{x}} \int_{0}^{\eta_{B}} f\left(Y_{t}\right) d t, \quad \tilde{x} \in \mathbb{R}^{d-1},
$$

for our isotropic stable process $Y_{t}$ in $\mathbb{R}^{d-1}$. Here, $B$ is the unit ball in $\mathbb{R}^{d-1}, \eta_{D}$ is the first exit time of $Y$ from $D \subset \mathbb{R}^{d-1}$, and $\mathcal{P}, \mathcal{E}$, are, respectively, the distribution and expectation corresponding to $Y$. For $r>0$, by scaling, $1 /\left(r^{-\alpha} \lambda_{1}\right)$ is the eigenvalue for $G_{r B}$ and $\mathcal{P}_{\tilde{x}}\left\{\eta_{r B}>t\right\} \leq c e^{-t r^{-\alpha} \lambda_{1}}, 0<t<\infty$. Let $\mathcal{C}$ be the cylinder as in (3). For $t>0$, fixed $x=\left(x_{1}, \tilde{x}\right) \in \mathcal{P}_{\beta}$ and $s \geq 1 \vee 2 x_{1}$, we have by Lemma 5 that

$$
\begin{aligned}
\mathbf{P}_{x}\left\{\tau_{\beta}>t\right\} & =\mathbf{P}_{x}\left\{\tau_{\beta}>t, \tau_{\beta}=\tau_{s}\right\}+\mathbf{P}_{x}\left\{\tau_{\beta}>t, \tau_{\beta}>\tau_{s}\right\} \\
& \leq \mathbf{P}_{x}\left\{\tau_{\mathcal{C}}>t\right\}+\mathbf{P}_{x}\left\{X_{\tau_{s}} \in \mathcal{P}_{\beta}\right\} \leq c e^{-t s^{-\alpha \beta} \lambda_{1}}+c s^{-\alpha \beta p_{0}} .
\end{aligned}
$$

Let us take $s=t^{(1-\epsilon) /(\alpha \beta)}$, where $0<\epsilon \leq 1 / 2$. We get

$$
\mathbf{P}_{x}\left\{\tau_{\beta}>t\right\} \leq c e^{-t^{\epsilon} \lambda_{1}}+c t^{-(1-\epsilon) p_{0}} \leq c t^{-(1-\epsilon) p_{0}}
$$

for large $t$. Thus, letting $p=(1-2 \epsilon) p_{0}$ we get

$$
\mathbf{E}_{x} \tau_{\beta}^{p}=\int_{0}^{\infty} p t^{p-1} \mathbf{P}_{x}\left\{\tau_{\beta}>t\right\} d t \leq \text { const. }+ \text { const. } \int_{1}^{\infty} t^{-\epsilon p_{0}-1} d t<\infty .
$$

This finishes the proof.

Proof of Theorem 1. The result follows from Lemma 2 and Lemma 6.

We conclude with three remarks.

Because of scaling of $\left\{X_{t}\right\}$, Theorem 1 holds with the same $p_{0}$ for the more general parabola-shaped regions of the form

$$
\left\{x=\left(x_{1}, \tilde{x}\right): x_{1}>0, \tilde{x} \in \mathbb{R}^{d-1},|\tilde{x}|<a x_{1}^{\beta}\right\},
$$

for any $0<a<\infty$. 
If $\beta \downarrow 0$, then $\mathcal{P}_{\beta}$ approaches an infinite cylinder, for which the exit time has exponential moments (compare the proof of Lemma 6).

The second endpoint $\beta \uparrow 1$ suggests studying the rate of convergence (to 1 ) of the critical exponent of integrability of the exit time from the right circular cone as the opening of the cone tends to 0 . A partial result in this direction is given in [13. 1] contains more information on our stable processes in cones and a hint on how to approach the problem.

\section{ACKNOWLEDGMENT}

The second author is grateful for the hospitality of the Departments of Statistics and Mathematics at Purdue University, where the paper was prepared in part. We are grateful to the referee for pointing out several corrections.

\section{REFERENCES}

[1] R. Bañuelos and K. Bogdan, Symmetric stable processes in cones, Potential Analysis, 21 (2004), 263-288. MR2075671

[2] R. Bañuelos and T. Carroll, Sharp Integrability for Brownian Motion in Parabola-shaped Regions (2003), J. Funct. Anal. 218 (2005), 219-253. MR2101220

[3] R. Bañuelos, R. D. DeBlassie and R. Smits, The first exit time of planar Brownian motion from the interior of a parabola, Ann. Prob. 29 (2001), 882-901. MR1849181(2002h:60165)

[4] R. Bañuelos and R. Smits, Brownian motion in cones, Prob. Th. Rel. Fields, 108 (1997), 299-319. MR1465162 (98k:60065)

[5] M. van den Berg, Subexponential behavior of the Dirichlet heat kernel, J. Funct. Anal. 198 (2003), 28-42. MR1962352 (2003m:60211)

[6] R. M. Blumenthal, R. K. Getoor and D.B. Ray, On the distribution of first hits for the symmetric stable process, Trans. Amer. Math. Soc. 99 (1961), 540-554. MR0126885 (23:A4179)

[7] K. Bogdan and T. Jakubowski, Problème de Dirichlet pour les fonctions $\alpha$-harmoniques sur les domaines coniques (2004), preprint.

[8] K. Bogdan and T. Żak, On Kelvin transformation, to appear in J. Theoretical Probability.

[9] K. Burdzy and T. Kulczycki, Stable processes have thorns, Ann. Probab. 31 (2003), 170-194. MR $1959790(2003 \mathrm{k}: 60079)$

[10] D. L. Burkholder, Exit times of Brownian motion, harmonic majorization, and Hardy spaces, Advances in Math. 26(2) (1977), 182-205. MR0474525 (57:14163)

[11] R. D. DeBlassie and R. Smits. Brownian motion in twisted domains, Transactions of the American Mathematical Society 357 (2005), 1245-1274. MR2110439

[12] R. DeBlassie, The first exit time of a two-dimensional symmetric stable process from a wedge, Ann. Prob. 18 (1990), 1034-1070. MR 1062058(91j:60078)

[13] T. Kulczycki, Exit time and Green function of cone for symmetric stable processes, Probab. Math. Statist. 19(2) (1999), 337-374. MR1750907 (2001f:60077)

[14] W. Li, The first exit time of Brownian motion from unbounded convex domains, Annals of Probability 31 (2003), 1078-1096. MR.1964959 (2004c:60126)

[15] M. Lifshitz and Z. Shi, The first exit time of Brownian motion from parabolic domain, Bernoulli 8(6) (2002), 745-767. MR1963660 (2004d:60213)

[16] P. J. Méndez-Hernández, Exit times from cones in $\mathbb{R}^{n}$ of symmetric stable processes Illinois J. Math. 46 (2002), 155-163. MR1936081 (2003i:60070)

[17] K.-I. Sato, Lévy Processes and Infinitely Divisible Distributions, University Press SpringerVerlag, Cambridge, 1999. MR 1739520 (2003b:60064)

Department of Mathematics, Purdue University, West Lafayette, Indiana 47907-1395

E-mail address: banuelos@math.purdue.edu

Institute of Mathematics, Polish Academy of Sciences, Poland - And - Institute of Mathematics, Wrocław University of Technology, 50-370 Wroceaw, Poland

E-mail address: bogdan@im.pwr.wroc.pl 